\title{
L'éducation en milieu rural : perceptions et réalités
}

Pierre-Louis Gauthier et Odile Luginbühl

\section{(2) OpenEdition}

Journals

Édition électronique

URL : https://journals.openedition.org/ries/2226

DOI : $10.4000 /$ ries. 2226

ISSN : 2261-4265

\section{Éditeur}

France Education international

\section{Édition imprimée}

Date de publication : 1 avril 2012

Pagination : 35-42

ISBN : 978-2-85420-594-7

ISSN : $1254-4590$

\section{Référence électronique}

Pierre-Louis Gauthier et Odile Luginbühl, «L'éducation en milieu rural : perceptions et réalités », Revue internationale d'éducation de Sèvres [En ligne], 59 | avril 2012, mis en ligne le 01 avril 2012, consulté le 21 septembre 2021. URL : http://journals.openedition.org/ries/2226 ; DOI : https://doi.org/10.4000/ ries.2226 


\title{
L'éducation en milieu rural : perceptions et réalités
}

\author{
Pierre-Louis Gauthier \\ Odile Luginbühl
}

La problématique de l'éducation en milieu rural est-elle anachronique? Dans le contexte contemporain de la mondialisation, les représentations habituellement associées au « rural » semblent renvoyer à un passé révolu, archaïque pour certains, nostalgique pour d'autres, où l'école garderait les contours figés d'une image d'Épinal. Pourtant, près de la moitié de la population mondiale vit encore dans des zones considérées comme rurales, ce qui permet d'affirmer l'actualité d'une réflexion sur l'éducation qui y est dispensée et justifie une analyse plus approfondie des réalités qui la caractérisent.

Certes, la notion de milieu rural, à l'échelle mondiale, recouvre des données géographiques, économiques et sociales fort différentes, et les perspectives ouvertes par ce dossier de la Revue internationale d'éducation éclairent la diversité, non de la ruralité, mais des ruralités qui conditionnent le fonctionnement des écoles rurales sur les cinq continents. À travers neuf articles émanant de chercheurs et d'acteurs de l'éducation et une riche bibliographie commentée, la complexité des situations est abordée dans ses dimensions structurelles, pédagogiques et économiques.

Cependant, au-delà des particularismes, ces articles témoignent d'un fait éducatif persistant dans tous les systèmes éducatifs et qui demeure le gage d'une possible égalité devant l'éducation. On sait que tout n'est pas comparable. Pourtant, une série d'invariants de la problématique de l'éducation en milieu rural ressort des études menées sur des milieux à la fois variés et univoques. En permettant de resituer ces invariants dans la logique d'un système, la contextualisation apportée par chaque auteur légitime les comparaisons effectuées au cœur du paradigme de l'éducation rurale. Les principaux paramètres qui la caractérisent et que le lecteur retrouvera au long des analyses du dossier sont présentés ici.

\section{L'IMPLOSION DE LA RURALITÉ DANS LE MONDE}

$\mathrm{Au}$ tournant du $\mathrm{XXI}^{\mathrm{e}}$ siècle, pour la première fois de son histoire, l'humanité voit les populations urbaines supplanter en nombre les populations rurales. Globalement, cinquante-trois pour cent des humains vivaient dans des 
villes en 2010. Soixante-quinze pour cent s'y seront établis en 2050. L'attractivité $\mathrm{du}$ mode de vie urbain en matière de production, de consommation, de culture et, surtout, de revenus bien supérieurs semble irrésistible. L'uniformisation des paysages urbains des grandes métropoles dans le monde symbolise cet essor inouï. La contrepartie est l'amoindrissement relatif du monde rural, qui continue néanmoins à alimenter les gigantesques migrations qui gonflent les villes et leurs territoires périurbains. Parmi les continents abordés dans le présent dossier, en Asie comme en Afrique, les populations rurales représentent de $63 \%$ à $70 \%$ de la population totale. Par contre, alors que l'Europe recense encore $26 \%$ de ruraux, le continent sud-américain a le plus faible taux de population rurale au monde, $21 \%$, avec cependant de grandes disparités entre les pays qui le composent ${ }^{1}$.

De très larges régions restent marquées par un mode de vie rural où les activités agricoles, pastorales ou forestières gardent leur vitalité. Les paysages façonnés par l'évolution des techniques culturales, l'habitat, la répartition des terres, la vie sociale, reproduisent dans leur extrême diversité l'allégeance de ces populations à la terre, à la forêt, à la montagne. Organisées en ruralités, elles sont assimilées à la tradition, voire à un certain passéisme souvent stigmatisé. Jugées pauvres, sans culture ni raffinement, les ruralités sont volontiers confondues avec le sous-équipement en matière de santé et d'éducation. L'homme rural est parfois doté d'un statut légal particulier, comme le hukou chinois évoqué par Xiaohui Wang, qui marque une sorte d'infériorité.

Au-delà des clichés réducteurs ou, au contraire, abusivement idéalisés selon une mode récente, il importe de cerner le concept de ruralité et la réalité $\mathrm{du}$ fait éducatif qui s’y attache.

Déterminer la ruralité par défaut ou en opposition à l'urbanité est tout à fait insuffisant. Les ruralités certes ont su préserver une identité forte, un genre de vie propre, liés au localisme. Mais elles participent également à des systèmes réticulaires multiples d'échanges, de communication, de production, de modélisation. Elles sont traversées par les courants de la mondialité, dont elles absorbent peu à peu les composants. On constate partout une sorte d'hybridation entre appartenance identitaire et flux mondialisés. C'est probablement ce qui distingue les ruralités des autres milieux. L'analyse que propose l'article de Laurent Rieutort, en ouverture du dossier, apporte un éclairage renouvelé sur la problématique rurale, cette « composition sociale du monde » selon l'expression de l'auteur, soumise à la périphérisation des activités humaines.

1. Données de la Banque mondiale, 2010. 


\section{LE CONCEPT DE RURALITÉ EN MATIÈRE D'ÉDUCATION}

La généralisation de l'éducation universelle est née au XIX ${ }^{\mathrm{e}}$ siècle en Europe et dans les pays sous influence occidentale comme l'Argentine, pays d'immigration qui a hérité d'une longue tradition scolaire, comme le montre Flavia Terigi. La création de l'école rurale répondait à une demande des classes populaires et à un souci d'égalité devant l'accès à l'instruction, dans des contextes sociétaux majoritairement ruraux. Elle a contribué à cimenter les unités nationales et elle reste - plusieurs articles du dossier le signalent porteuse de l'idéologie politique du régime qui la promeut : idéal républicain en France, socialiste en Tanzanie, égalitariste en Australie, communiste en Chine.

Elle a, en même temps, accompagné les progrès de la révolution agricole avec la vulgarisation des techniques nouvelles et des engrais. Dans les villages des pays occidentaux, la maison d'école, le maître d'école symbolisent bien ce passé. L'école rurale dans le monde reste marquée par l'antériorité du modèle de l'école occidentale.

L'éducation participe étroitement au paradigme de la ruralité. Marqueur efficace, elle témoigne des permanences et des évolutions des sociétés rurales, dont le fondement est historiquement lié à l'agriculture. Partout présent dans les sociétés rurales, le fait éducatif reflète la vigueur ou la décadence de ces communautés. Par exemple, on sait combien le développement de la santé est étroitement lié à la solidité et à la durabilité du fait éducatif. De même, dans un contexte de concurrence qui affaiblit l'agriculture, la vie économique rurale passe par l'école, qu'il s'agisse de l'évolution des techniques agro-pastorales ou des activités artisanales et commerciales qui dépendent du maintien d'un groupe social structuré. C'est le cas, notamment, en Tanzanie, où la scolarisation des Massaï est présentée par Nathalie Bonini comme une réponse à la précarité née de la dégradation de leurs pâturages.

Parmi les invariants de l'éducation en milieu rural se pose le lien qu'elle entretient avec le milieu local. L'éducation se fond-elle en symbiose dans le milieu rural ou est-elle une institution à part, créée et gérée par un pouvoir distant et lointain, parfois en opposition à la ruralité, ainsi que le rappelle Yves Alpe ? Se présente-t-elle comme une rémanence identitaire liée aux cultures locales ou régionales ou participe-t-elle d'un dessein d'unité nationale ? Est-elle vouée aux mutations liées à la périphérisation des activités humaines ou conserve-t-elle des spécificités irréductibles?

L'isolement, corollaire de l'éloignement, a incité à puiser dans l'environnement social et culturel les ressources nécessaires à cette activité intellectuelle souvent unique dans le village qu'est l'éducation. Dans tous les pays, le rôle de l'environnement social et culturel des communautés locales est fondamental dans le maintien des spécificités culturelles et éducatives. En Finlande, 
par exemple, où $20 \%$ des petites écoles sont en zone rurale, Arja-Sisko Holappa souligne combien l'identité culturelle est entretenue par les communautés qui entourent l'école d'un environnement « protecteur».

Cependant, si le localisme favorise la sociabilité et la proximité, il peut signifier aussi le renfermement communautaire. Dans beaucoup de régions rurales, la défaillance du pouvoir central a depuis longtemps incité les collectifs de parents à prendre en main la construction et la gestion de l'école, imprimant ainsi la culture locale dans l'action proprement scolaire. Cette intervention ne va pas sans sacrifices financiers, comme l'indiquent aussi bien Nathalie Bonini pour la Tanzanie que Xiaohui Wang pour la Chine.

Le fait éducatif en milieu rural est, comme tout son environnement, marqué par l'étendue, les distances parfois longues, l'éloignement des centralités urbaines, des lieux de décision et de financement. Il est maximum sur des territoires vastes, à densités très faibles voire nulles. La description que donne William Newman du Territoire du Nord en Australie, autour d'Alice Springs, en est l'exemple type.

La question de l'éloignement doit cependant être modulée, car des réponses structurelles peuvent être apportées, notamment par l'organisation en constellation autour de centralités secondaires qui prennent en quelque sorte le relais de l'espace villageois. Le fonctionnement en constellation se vérifie particulièrement pour l'enseignement secondaire, avec les rayons à plus grande courbure pour certaines options et pour l'enseignement supérieur. Cette organisation étoilée est favorable à la décentralisation de l'organisation et du financement que l'on distinguera soigneusement des pratiques d'enseignement. Les différents dispositifs de modulation de l'espace éducatif rural par des regroupements, tels que les décrit Yves Alpe, sont peu connus et mériteraient une évaluation fine qui n'existe pas.

\section{Atouts et falblesses DE L'ÉCOLE RURALE}

Le phénomène de fort développement urbain qui concerne désormais toutes les régions du monde a entraîné un renversement du modèle de référence des systèmes éducatifs. Prenant le cas de l'Argentine, Flavia Terigi montre que les politiques éducatives, comme la recherche en éducation, se sont développées principalement en fonction des écoles urbaines, ce qui a entraîné une marginalisation des écoles rurales. En d'autres termes, la spécificité des problèmes qui se posent dans les écoles rurales n'est que ponctuellement prise en compte et, le plus souvent, pour les rapporter au modèle urbain - comparaisons qui sont en général à leur détriment.

Le milieu rural peut cependant réserver à l'éducation des atouts favorables en termes de socialisation, comme on l'a vu, mais aussi d'efficacité pédagogique. Les analyses d'Arja-Sisko Holappa en Finlande et d'Yves Alpe en France 
montrent que les conditions sont parfois plus favorables qu'en milieu urbain, le relationnel plus stable. La connaissance de l'environnement humain, la moindre nécessité de pallier les problèmes de discipline, les effectifs réduits, militent en faveur de résultats équivalents sinon supérieurs en milieu rural, au moins pour l'enseignement primaire.

Pour répondre aux besoins propres à l'éducation dans le milieu rural, la pédagogie a cherché à créer des procédures adaptées à ses publics et inventé des stratégies locales. Le développement des nouvelles technologies, en particulier Internet et les réseaux sociaux, permet de remédier à l'enclavement. Sur de vastes étendues peu peuplées, avec un habitat très dispersé, on trouvait jusqu'à maintenant peu de solutions éducatives, hormis le regroupement en internat dans quelques agglomérations centrales, jusqu'à l'avènement des techniques nouvelles d'information et de communication. William Newman donne, pour le Territoire du Nord australien, un exemple d'utilisation de l'informatique, au prix d'un investissement disproportionné avec la population concernée, il est vrai.

Dans le cône sud de l'Amérique, Flavia Terigi montre comment les pédagogues argentins, s'affranchissant de cadres programmatiques émanant du «centre » et conçus pour les écoles urbaines, ont élaboré des méthodes, un « faire » original dont l'efficacité s'est révélée assez évidente pour qu'on envisage de les transférer en ville, lorsque l'enseignement s'effectue dans un contexte de regroupements d'élèves (par exemple, dans le cadre des actions de réinsertion scolaire) proche des conditions des écoles rurales. Le maintien des classes multigrades ou des classes uniques en France est l'une de ces originalités pédagogiques de l'éducation en milieu rural. Elles répondent à la diversité mieux que les classes à double flux.

Dans les pays multilingues, les langues jouent un rôle essentiel, dans un double mouvement : d'une part, un lien d'appartenance forte parmi les facteurs de la rémanence culturelle, d'autre part, un mode d'intégration nationale.

L'Afrique voit actuellement l'émergence des langues dites « nationales », dans le contexte postcolonial. Ainsi, Afsata Paré/Kaboré rappelle les étapes de la mise en place de programmes de scolarisation bilingues en régions rurales introduisant, auprès du français, l'une des huit langues nationales africaines du Burkina-Faso. Leur efficience dans l'amélioration de la qualité des apprentissages incite maintenant à leur généralisation. Là encore, les projets lancés en milieu rural jouent en quelque sorte un rôle expérimental. En Tanzanie, c'est également une procédure de bilinguisme qui favorise la scolarisation des Massaï, toutefois selon une démarche différente décrite par Nathalie Bonini : il s'agit de faciliter l'accès de cette population, de langue maternelle maa, à la langue nationale, le swahili. Mais on voit poindre aussi l'une des limites de l'émancipation dans le cadre d'une éducation communautaire limitée aux langues locales ou nationales, les langues internationales ne prenant le relais que dans l'enseignement secondaire supérieur et l'enseignement supérieur. 
Ces différentes expérimentations montrent qu'un modèle de l'éducation rurale existe, mais qu'il nécessite une réflexion pédagogique et une compétence professionnelle spécifiques, comme le soulignent plusieurs articles du dossier. Se pose alors la question de la formation et du recrutement des enseignants - deux problèmes majeurs et étroitement associés de l'enseignement en milieu rural. Dans tous les pays, on observe des difficultés à recruter des enseignants titulaires, c'est-à-dire disposant d'une formation initiale validée, sur des postes dans les zones isolées. En outre, cette formation n’inclut pas de préparation pédagogique à l'enseignement dans les classes multigrades et les conditions d'isolement ne permettent guère les échanges entre enseignants ni leur participation à une formation continue.

\section{LE COÛT DES ÉCOLES RURALES}

Le coût de l'éducation en milieu rural est plus élevé qu'en milieu urbain. On évalue, pour la Finlande, le différentiel à $+14 \%$. Quel que soit le niveau économique du pays, cette donnée ne peut être négligée. Les choix financiers sont toujours difficiles et les arbitrages litigieux. Quelle est la limite de maintien d'une école rurale? Quels sont les critères de rentabilité, dirait-on en langage néolibéral ? Dans les régions reculées, le rural isolé génère des frais supplémentaires de transport, d'hébergement, de déplacement des personnels et des élèves. Prenant prétexte de leurs déficits, maints pays se posent la question du maintien de l'éducation en milieu rural et inclinent parfois à des coupes drastiques, au risque d'affaiblir leur propre système.

Plusieurs articles du dossier montrent cependant que, selon des degrés divers, les pays occidentaux continuent à investir dans l'école rurale. L'éducation dans les régions rurales françaises maintient, grâce à des moyens importants provenant des collectivités et de l'État, un bon niveau de formation. Le meilleur élève des évaluations PISA, la Finlande, reste très attaché à ses écoles rurales, assuré que le respect de l'égalité de tous devant l'éducation est le gage d'un développement à terme de l'économie et du maintien de la cohésion sociale. Le Territoire du Nord en Australie, en maintenant pour cent trente élèves un système coûteux de télé-enseignement, est un autre exemple.

La situation est fort différente dans les pays en voie de développement ou émergents, où la disparité entre les milieux ruraux et urbains est criante, à l'exemple de la Chine et de la Tanzanie. Le niveau d'investissement dans l'éducation des pays en développement reste faible, sinon nul. Les populations sont mises à contribution sous la forme de l'impôt scolaire, qu'il s'agisse de payer les personnels ou de construire des écoles communautaires. Plus que la surpopulation scolaire, la pénurie des enseignants contraints à un double métier ou choisissant de migrer vers les villes, accentue l'échec de la scolarisation universelle. En face d'une augmentation générale de la scolarisation, le souséquipement des écoles africaines en brousse reste chronique. Les fondamentaux 
que sont l'eau potable, l'électricité, la clôture, les dortoirs et les routes d'accès à l'école sont absents. L'absence de dispensaire compromet le couple santééducation. Il n'est pas certain que les efforts consentis par les populations aboutissent à des améliorations durables, tant les besoins sont immenses. En Tanzanie, par exemple, Nathalie Bonini montre que l'émancipation sociale par l'école est compromise pour les habitants des zones rurales isolées.

C'est sans doute sur ces considérations économiques que se fonde la stigmatisation de l'école rurale que l'on retrouve dans tous les pays en voie de développement ou émergents. La pauvreté du système, le retard des réformes comme l'obligation et la gratuité scolaires, l'absence de moyens, entraînent un nombre important de constats négatifs. Les rendements, le taux de survie sont faibles. Les taux d'abandon, de déperdition sont élevés. L'accès au niveau secondaire reste limité et infime au niveau supérieur. Le ratio filles-garçons ne se réduit pas. La qualification des enseignants demeure très faible. Le taux d'alphabétisation des adultes peine à s'élever.

\section{De nouveaux espaces D'ÉDUCATION}

L'accroissement des échanges et des migrations périphériques, internes et étrangères, entraîne une redéfinition des espaces de l'éducation. Dans les pays à forte croissance, les migrations vers les centralités urbaines atteignent des niveaux gigantesques entraînant, avec les adultes, des foules d'enfants d'origine rurale. En 2012, ces migrations en Chine atteindront 180 millions d'individus, soit trois fois la population de la France. L'analyse de Xiaohui Wang en tire les conséquences avec lucidité : la non-scolarisation de ces migrants par les autorités municipales urbaines, pour des raisons à la fois économiques et sociales, entraîne une scolarisation au rabais, souvent à la périphérie et dans des conditions matérielles dégradées.

L'Occident connaît une situation semblable, avec le gonflement des effectifs scolaires en périphérie urbaine et la discrète migration interne de catégories sociales plus favorisées, y compris les enseignants plus qualifiés, vers les établissements de centres urbains. Inversement se créent dans les couronnes urbaines concentriques des espaces dont la délimitation devient floue. Entre espaces suburbains et espaces ruraux existent des zones interstitielles qui empruntent des caractéristiques aux uns et aux autres, sans toutefois se confondre avec eux. On est souvent tenté de leur donner un statut éducatif particulier (écoles de migrants en Chine), comme s'ils échappaient au droit commun.

En sens inverse, la mobilité autorise des migrations vers les régions rurales. Le télétravail, notamment dans le tertiaire, et les transports rapides autorisent dorénavant ce genre de mutations qui cherchent à rendre compatible emploi et genre de vie. 
Ces populations nouvelles de "néo-ruraux », évoquées par Yves Alpe, importent avec elles de nouvelles exigences en matière d'éducation et d'environnement culturel, auxquelles l'école rurale se doit de répondre. Elles importent également la mobilité et leurs stratégies d'évitement. Là aussi, la modélisation est à l'œuvre.

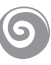

Ce dossier présente la complexité des situations que recouvre le terme d'éducation en milieu rural. Tous les articles évoquent des difficultés d'organisation structurelles et matérielles, dues à l'éloignement géographique des grands centres urbains, à la diminution des populations concernées et aux surcoûts entraînés, mais tous soulignent les enjeux sociétaux de l'école rurale, comme lien social et gage de l'égalité devant l'accès à l'enseignement.

Les réponses des politiques éducatives diffèrent selon qu'elles s'efforcent d'équilibrer la tension entre ces deux pôles, en assumant au niveau national la spécificité de ce secteur de l'enseignement, ou qu'elles délèguent ce rôle aux populations locales, les contraintes économiques l'emportant sur la fonction sociale.

Pourtant, plusieurs expériences montrent que, précisément, les conditions particulières de l'enseignement en milieu rural ont suscité des démarches innovantes transférables à l'ensemble du système éducatif : pédagogie des petits groupes, utilisation des nouvelles technologies, enseignement bilingue, articulation à l'économie locale, mise en réseau des établissements - en un mot «l'invention du faire», pour reprendre l'heureuse expression de Flavia Terigi. Ne peut-on alors reconsidérer la place de l'éducation en milieu rural, et faire de sa particularité, non pas un motif de stigmatisation, comme on l'a vu, mais un atout de la recherche pour l'amélioration de la qualité de l'éducation? 